\title{
Realization of mechanical rotation in superfluid helium
}

\author{
E.B. Gordon ${ }^{1}$, M.I. Kulish ${ }^{1}$, A.V. Karabulin ${ }^{2}$, V.I. Matyushenko ${ }^{3}$, E.V. Dyatlova ${ }^{1,4}$, \\ A.S. Gordienko ${ }^{1,4}$, and M.E. Stepanov ${ }^{1,4}$ \\ ${ }^{1}$ Institute of Problems of Chemical Physics RAS \\ 1 Akad. Semenov Ave., Chernogolovka, Moscow region 142432, Russia \\ E-mail: gordon.eb@gmail.com \\ ${ }^{2}$ National Research Nuclear University MEPhI (Moscow Engineering Physics Institute) \\ 31 Kashirskoe Shosse, Moscow 115409, Russia \\ ${ }^{3}$ The Branch of Talrose Institute for Energy Problems of Chemical Physics RAS \\ 1 Akad. Semenov Ave., Chernogolovka, Moscow region 142432, Russia \\ ${ }^{4}$ Lomonosov Moscow State University, 1 Leninskie Gory, Moscow 119991, Russia
}

Received December 13, 2016, published online July 25, 2017

\begin{abstract}
The possibility of using miniaturized low-power electric motors submerged in superfluid helium for organization of rotation inside a cryostat has been investigated. It has been revealed that many of commercial micromotors can operate in liquid helium consuming low power. Turret with 5 sample holders, assembled on the base of stepper motor, has been successfully tested in experiments on the nanowire production in quantized vortices of superfluid helium. Application of the stepper motor made it possible in a single experiment to study the effect of various experimental parameters on the yield and quality of the nanowires. The promises for continuous fast rotation of the bath filled by superfluid helium by using high-speed brushless micromotor were outlined and tested. Being realized, this approach will open new possibility to study the guest particles interaction with the array of parallel linear vortices in He II.
\end{abstract}

PACS: $67.25 . d k$ Vortices and turbulence;

07.20.Mc Cryogenics; refrigerators, low-temperature detectors, and other low-temperature equipment.

Keywords: superfluid helium, rotation, laser ablation.

\section{Introduction}

It is required for a number of low-temperature experiments to carry out some kinds of movements and even a rotation of different elements of devices inside a cryostat. Such procedures are technically difficult to perform in the cryostats with liquid helium used as a coolant. And it is especially difficult in experiments with superfluid helium, where the transition to the superfluid state is achieved by pumping helium vapor out of the cryostat to lower the temperature below $\lambda$ point $\left(T_{\lambda}=2.17 \mathrm{~K}\right)$. It is connected with the fact that additionally to the requirements of minimization of heat flux from outside and provision of the device operability at low temperatures one could ensure that the device can operate in a vacuum (at typical experiment temperatures $T=1.5 \mathrm{~K}$ helium vapor pressure is $470 \mathrm{~Pa}$ ).

The transfer of motion from outside, for instance, through a cryostat cap by, for example, Wilson seals or magnetic coupling in some cases cannot provide the required speeds of rotation. In addition, a mechanical tool that transmits rotation from the "warm" cap to liquid helium, is simultaneously a heat conductor, induced additional evaporation of liquid helium. It should be noted that for the rotation of a bath with superfluid helium in order to organize inside of the He II an array of parallel quantized vortices [1] it was not invented anything better than to rotate the heavy and bulky cryostat as a whole; considering the problems of switching both electrical circuits and vacuum pumping devices during the rotation to be easier.

We were faced with similar problems while improving the method of nanowires synthesis in quantized vortices of superfluid helium [2,3]. The ablation from the surface of immersed in superfluid helium metallic targets by focused beam of pulse-repetition laser with sub-nanosecond pulse duration was found to be a convenient way of metal atoms and small clusters introduction into superfluid helium [4]. 
Pulsed laser ablation (PLA) in a liquid is commonly used as a method of nanomaterial production [5-7]. Its main advantages are the universality (nanoparticles of different materials may be produced with the same technique), the compactness of a reactor as well as the high chemical purity of the product (because no precursors stabilizers or other chemicals are used in the synthesis). The latter is especially important because a number of important applications such as medicine [8,9], catalysis $[10,11]$, sensors $[12,13]$, etc. require nanoparticles with an ultra clean surface.

One of the difficulties of PLA application for production of significant amounts of a nanomaterial is a perishing of the target surface during deposition. A crater is formed on the surface of target in a focus of repetitive laser pulses and its depth progressively grows from pulse to pulse. The corresponding increasing of the target's surface area (with which laser radiation interacts) leads to the reduction of the density of absorbed energy, which by-turn results in a weakening of the ablation until its complete termination. The standard technique used for preventing a deep crater formation on the surface of the target is its rotation $[14,15]$. Typically, the target rotation does not cause any technical difficulties, as the PLA commonly realized at ambient conditions, and the water or some alcohol is used as a liquid. However, in our case it is necessary to organize the rotation inside the cryostat with superfluid helium. To date we have used an alternative approach of scanning the laser beam along the surface of immovable target [16]. But because of the changes in the geometrical position of the ablation place on the surface of the target this approach excludes application of the optical methods for monitoring and studying the condensation process.

Currently, the compact and low-energy consuming electric micromotors, which in principle could be operable in liquid helium, have appeared. An idea to use electric motor for organization of rotation in superfluid helium is not new [17]. Several research groups at different times used this method for the production of various low-temperature experiments. Initially home-made motors with a superconducting coil were used. The complexity of the manufacture of such motors of small size imposes a limitation on the distribution of this technique. Therefore, different groups have been attempted to use commercially available solutions. It turned out that it is possible to use commerciallymanufactured motors after minor modifications to helium experiments. In several studies [18-20] electric motors were used stepper to rotate the sample at a predetermined angle. In a recent paper [21] on the study of laser ablation in superfluid helium electric motor were used to rotate the target. The motor was mounted outside the liquid in the helium vapor, at the top part of cryostat, and the connection to the target was carried out by a shaft. The speed of rotation of target was only $3 \mathrm{rpm}$.
This paper is devoted to the problems of using such motors immersed in He II to sustain the motion of various types in superfluid helium.

\section{The problem formulation}

From the technical point of view, submerging of the micromotor into superfluid helium would be the best solution, but it imposes the strict conditions on the power that a motor releases in liquid helium. The resulting heat causes an additional helium evaporation and (at given rate of pumping) an increase of liquid helium steady temperature (this effect is especially pronounced for helium because its heat of vaporization consists only in $5 \mathrm{~K}$ per atom). It should be emphasized that enough high heat flow could result in liquid helium temperature increasing above $\lambda$ point, where both superfluidity and quantized vortices don't exist and thus the formation of nanowires is impossible.

It is easy to calculate that the dissipation in the He II of power of $1 \mathrm{~W}$ leads to evaporation of $1.4 \cdot 10^{22}$ helium atoms, i.e., $0.5 \mathrm{~cm}^{3}$ of liquid helium, per second. Total volume of superfluid helium in our experiments is about $500 \mathrm{~cm}^{3}$, it means that with such heat release an experiment may last only $1000 \mathrm{~s}$, or less than $17 \mathrm{~min}$.

In addition, in such conditions to achieve the pressure equal to $80 \mathrm{~Pa}$ (corresponding to the liquid helium temperature $1.2 \mathrm{~K}$ ) one should pump helium vapor with rate of 500 liters of room temperature gas per second, which is possible only with the use of powerful and expensive mechanical pumps. With the typical for low-temperature experiment pumping rate of $25 \mathrm{l} / \mathrm{s}$ one should work at such heat release at a temperature as high as $2 \mathrm{~K}$, which is very close to the $\lambda$ point temperature.

The recent progress in miniaturization of electronics and in particular of the electric motors have made it possible to test the low-power electric motors immersed in superfluid helium for the target rotation. Of course, these motors are not designed for work in such extreme conditions, therefore, first and foremost, it was to find out whether they can generally operate at low temperatures inside superfluid helium and, if so, then how long it will be possible for them to rotate.

In the case of success, such a design solution could be useful for another, specific for superfluid helium important task. As is known, rotation of superfluid helium as a whole leads to the appearance of the array of parallel to the rotation axis quantized vortices, which density is proportional to the angular velocity (Feynman rule). It has been already mentioned that for experimental observation of this effect and for researching of quantized vortices properties the whole cryostat used to set in rotation together with all its electrical and vacuum connections. Naturally, the speed of such rotation should not exceed a circle per few seconds $[22,23]$. 
In addition, in our previous studies [2,24,25] the quantized vortices were nucleated by laser pulses in the focal spot on the target surface, i.e., in the same place and at the same time as the metal particles were embedded. On the one hand, it increases the efficiency of the vortex application as a template for the growth of nanowires in their core, but on the other hand, it made impossible to optimize the relative concentrations of metal and vortices - if one changes the energy of the laser pulse, both concentrations will change simultaneously. To solve this problem it was necessary to be able to change the density of vortices independently, which can be achieved by rotation of superfluid helium. Moreover, it seems attractive to produce the vortices parallel to each other hoping to form longer nanowires.

With motor inside, one can rotate not cryostat as a whole but only a cell filled with superfluid helium (see Fig. 1(a)). Passing through the transparent walls of the cell the laser light will focus on the outer surface of the cylinder located on the motor shaft, which will simultaneously be the target for ablation. However it will be sufficient to rotate only the central cylinder (see Fig.1(b)). Indeed, accordingly to our recent research [26], the whole process of a metal condensation in He II occurs at distances less than one millimeter from the laser spot. The field of velocities of the liquid just near the target should be cylindrically symmetrical and close to that in the rotating cryostat, and provided the rotation is sufficiently fast the number of vortices generated by the rotation in the region adjacent to the cylinder surface should exceed the number of vortices generated by the laser pulse.

Thus, to formulate the requirements for the electric motor it is necessary, first of all, to determine how fast it should rotate. The density of the vortex lines $L$ in our experiments is typically about $10^{7} \mathrm{~cm}^{-2}$ [4]. Accordingly to Ref. 27 this density corresponds to a certain velocity of the normal component $v_{n}$ :

$$
v_{n}=\frac{\sqrt{L}}{\gamma} \frac{\rho_{s}}{\rho},
$$

where $\gamma$ is the empirical coefficient, $\gamma=130, \rho$ and $\rho_{s}$ are the densities of liquid helium and its superfluid compo-

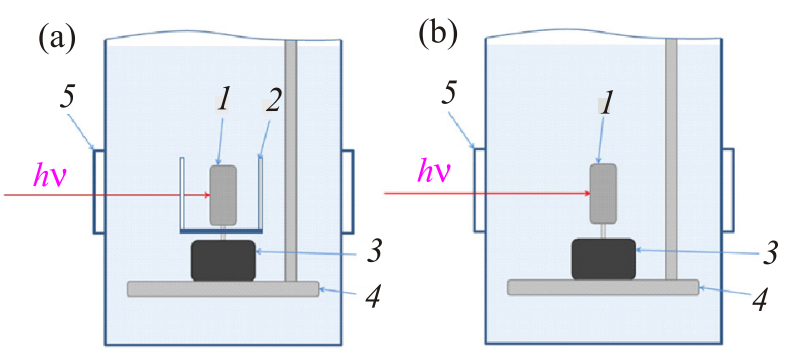

Fig. 1. Scheme of the liquid-helium cryostat (the laser beam is shown by arrow): with cell (a), without cell (b). 1 - cylindrical target; 2 - cell with transparent sidewalls; 3 - electromotor; 4 - support, 5 - sapphire window. nents, respectively. At $T=1.6 \mathrm{~K}, \rho_{s} / \rho=0.84$ [28] and in accordance with (1) the velocity of normal component in our experiment is equal to $20 \mathrm{~cm} / \mathrm{s}$.

On the other hand, in steady conditions, the velocity of normal component in proximity to the surface of the rotating cylinder with radius $R$ is equal to the linear velocity of the cylinder rotation:

$$
v_{n}=\omega R
$$

where $\omega$ is the angular velocity of the cylinder, $R$ is its radius (as accepted $R=1 \mathrm{~cm}$ ). Substituting corresponding values, one can find that the density of vortices formed by the rotation of the cylinder exceeds their density which is created by the laser pulse interaction with target surface at pulse repetition rate of $4 \mathrm{kHz}$, when the cylinder rotation frequency $f=\omega / 2 \pi$ is more than 20 circles per second.

The usage of miniature electric motor, namely, the step motor, embedded in superfluid helium, could solve another experimental problem. It would allow preparing in single low-temperature experiment several specimens, corresponding to different conditions (temperature, frequency of laser pulses and their energy, size of focusing spot, etc.). This promises to reduce by several times the number of experiments necessary to study the effects of various experimental parameters on the yield and quality of nanowires produced in superfluid helium.

The study of morphology and structures of nanowires was usually performed at room temperature by using the transmission electron microscopy and the energy-dispersive x-ray analysis. For that the nanowires were deposited on special substrates placed at the bottom of an experimental cell. Only after warming the cryostat up to room temperature the samples were transferred, for instance, to vacuum chamber of the electron microscope. Just one type of samples may be created in such experimental conditions, otherwise we will deal with the mixture of nanowebs prepared in different conditions or made of different metals. The low-temperature experiment, including the cooling and warming stages, lasts about four days, so the preparation of several specimens per one experiment means significant acceleration of an investigation. Such a way the simple operation of successive temporary uncovering the different supports for the nanoweb sedimentation with the aid of a step motor may significantly accelerate the study.

Since the operation of support exchange takes only few second the power restrictions for this step-like motion are much softer than for continuous rotation.

Although low-power motors have been used for rotation in superfluid helium, they were used only for turning the sample on the particular angle or for target rotation at low speed. This paper discusses a much more widespread use of these devices, including the organization of an ordered lattice of quantized vortices. 


\section{Design, fabrication and testing devices for transmitting rotation to the cryostat}

A manufactory's oil lubrication used in all motors initially under cooling to helium temperatures should freeze and such prevent rotation there. Because of this fact primarily, the oil was removed by motor rotation in ethanol for the time of 2-4 h. After drying the solid lubricant, molybdenum disulfide $\left(\mathrm{MoS}_{2}\right)$ was added through the holes of motor. Then the performance capabilities of the motors were tested in liquid nitrogen at $77 \mathrm{~K}$.

\subsection{Samples replacement}

The most simple was to realize the samples remote exchange in cryostat. In this case two-phase four-wire stepper micromotor was chosen as electromotor (Fig. 2) [29]. The angle of its rotation per one step was $18^{\circ}$.

The important feature of this motor was the absence of brushes and in such a manner there was no problem of the contact of brushes with collector of the motor at low temperatures when the solid lubricant - molybdenum disulfide - becomes insulator.

The motor full $360^{\circ}$ revolution corresponded to 20 steps. Taking into account geometric sizes of lowtemperature cell and the diameter of standard grids for TEM (3 mm) the turret with five grids was found to be optimal. In this case the turret should rotate by $72^{\circ}$ which corresponds to the 4 motor steps. In other words, for the operation of sample exchange 4 electrical pulses should be applied to the motor.

The stepper motor (SM) self-made control circuit is presented in Fig. 3. It consists of two microcontrollers

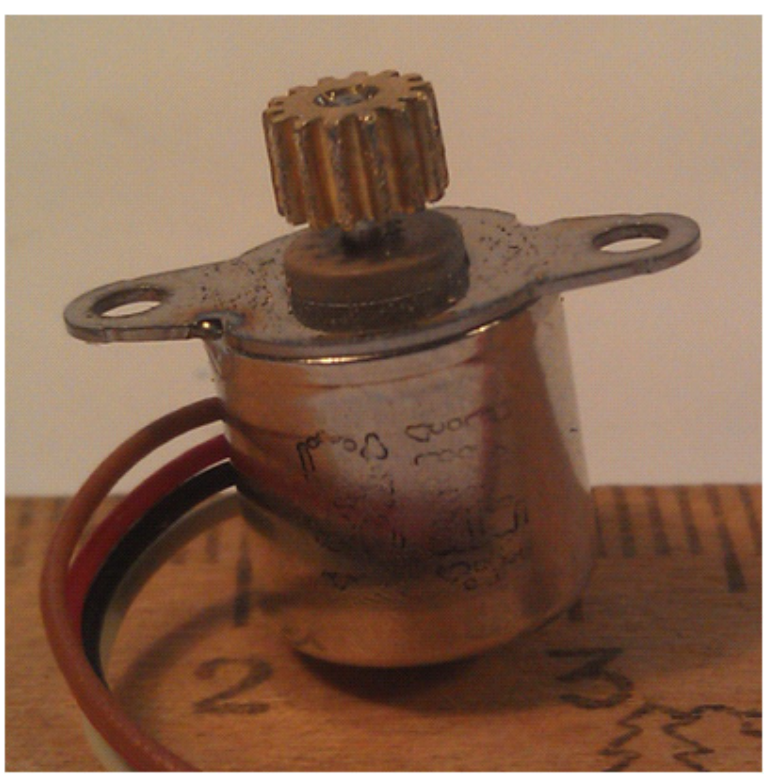

Fig. 2. The photo of step micromotor used.
A3967SLB, PIC12F675 and provides tuning of such parameters as the rotation direction, the frequency of step pulses and the mean current of SM windings. The controller PIC12F675 handles with using the switch Sw. In middle position of the switch the current in both windings is absent.

The mechanical part of the sample exchange tool is presented on Fig. 4.

The stepper motor 1 is attached vertically to the bottom of support 2. The turret 3 with five housings for the grids is tightly fixed on the motor axis. The disk 4 fitted with the openings which are $0.2 \mathrm{~mm}$ smaller then grids diameter (for prevention of grid spontaneous dropping out) is fixed on the top of the turret. The cap 5 with slit covers the turret from the top. The target 6 (indium foil in the photo) is placed directly above the slit. In any working position of the setup only one grid, that disposed in laser focus is open for deposition of the falling nanowires.

Visual control of the turret position is realized by using the slit in the cap by observation turret position through optical sapphire window of cryostat.

For reducing the heat release in the liquid helium the electric power is off during the most of the time, and thus there is no force holding the turret at a predetermined position. Fortunately, it turned out that stop-position is stable.

In our experiment, the stepper motor was to be activated only 4 times, and for a short time. Knowing the value of the jump in gas helium steady residual pressure at the moment when the motor acts, we estimated the thermal power during the operation of the electric motor in He II. At the motor rate of rotation of 1 step per second, the estimation gives $0.1-0.2 \mathrm{~W}$. This means that whole sample exchange operation during the experiment «costs» less than $1 \mathrm{~J}$; these losses are small enough not only for our problem, but for the most of the experiments carried out with liquid helium.

Testing of the technique was conducted in experiments aimed at revealing the dependence of morphology of nanostructures produced by laser ablation of indium in superfluid helium on the size of laser spot on the target at a constant pulse energy.

Our experimental setup has been described elsewhere [30]. It was assembled on the basis of the pumping helium optical cryostat. The solid state Nd: LSB diode-pumped laser used for metal ablation, has the following characteristics: pulse duration $0.4 \mathrm{~ns}$, pulse repetition rate $0.5-4 \mathrm{kHz}$, pulse energy $0.1 \mathrm{~mJ}$ and light wavelength $1.062 \mu \mathrm{m}$. Laser radiation had entered the cryostat through the sapphire windows and then it was focused on the target submerged in superfluid helium; the minimal diameter of focused laser spot was about $100 \mu \mathrm{m}$. The morphology and structure of ablation products were examined by transmission electron microscope JEM2100 company JEOL after a cryostat warming to the room temperature. 


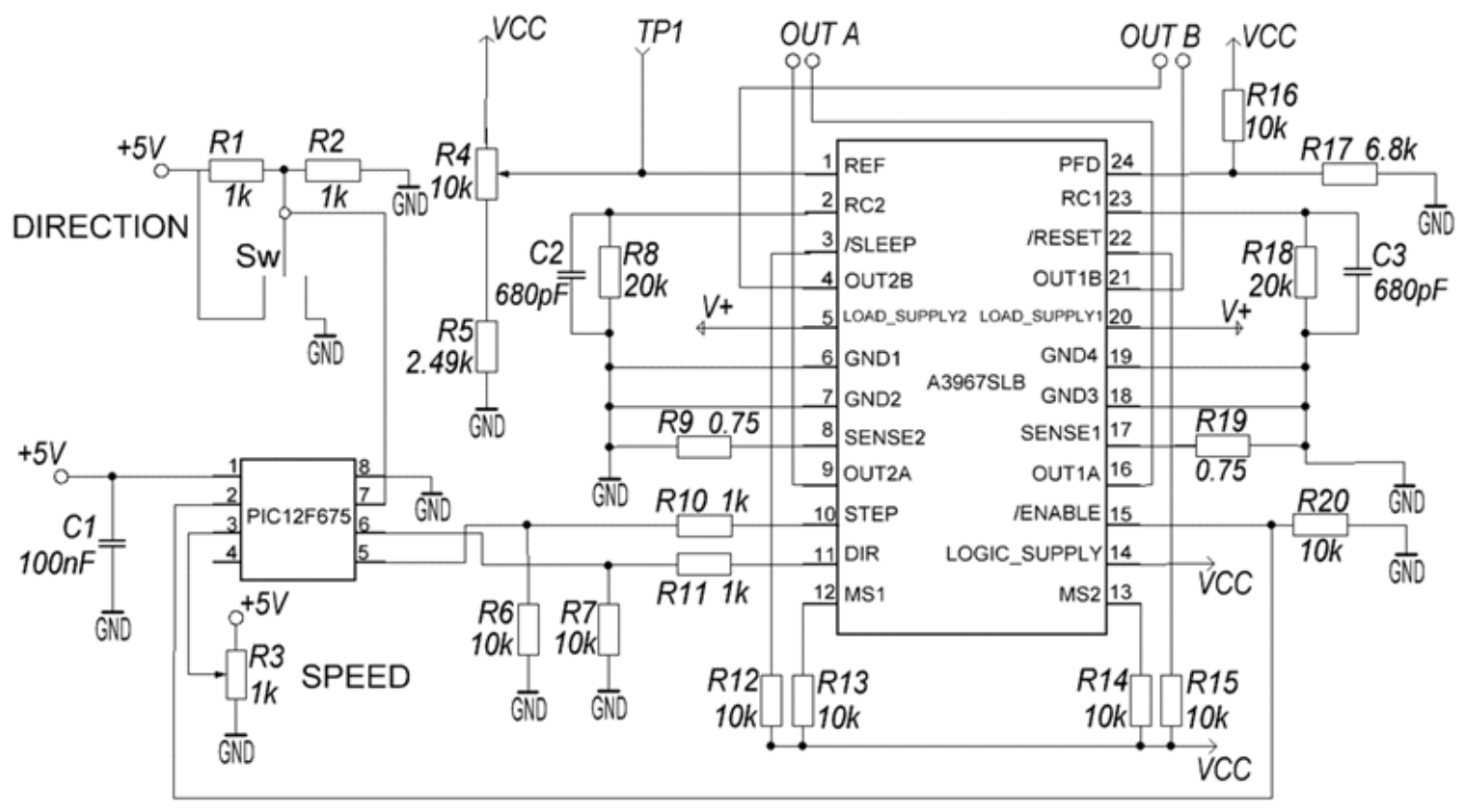

Fig. 3. The stepper motor control circuit.

Desired product was a metal nanowire, but along with them the spherical particles with an atomically smooth surface were observed. Their sizes were ranged from a few nanometers to several microns. The regular shape of spheres and their smooth surface left no doubt that as nanowires [30] the spheres were formed from the molten clusters. We thought that they had formed at high metal densities in superfluid helium when molten clusters collided with each other not having enough time for cooling [24,30]. There is, however, an alternative explanation, according to which clusters were not formed in liquid helium, but they were the products of the ablation process.
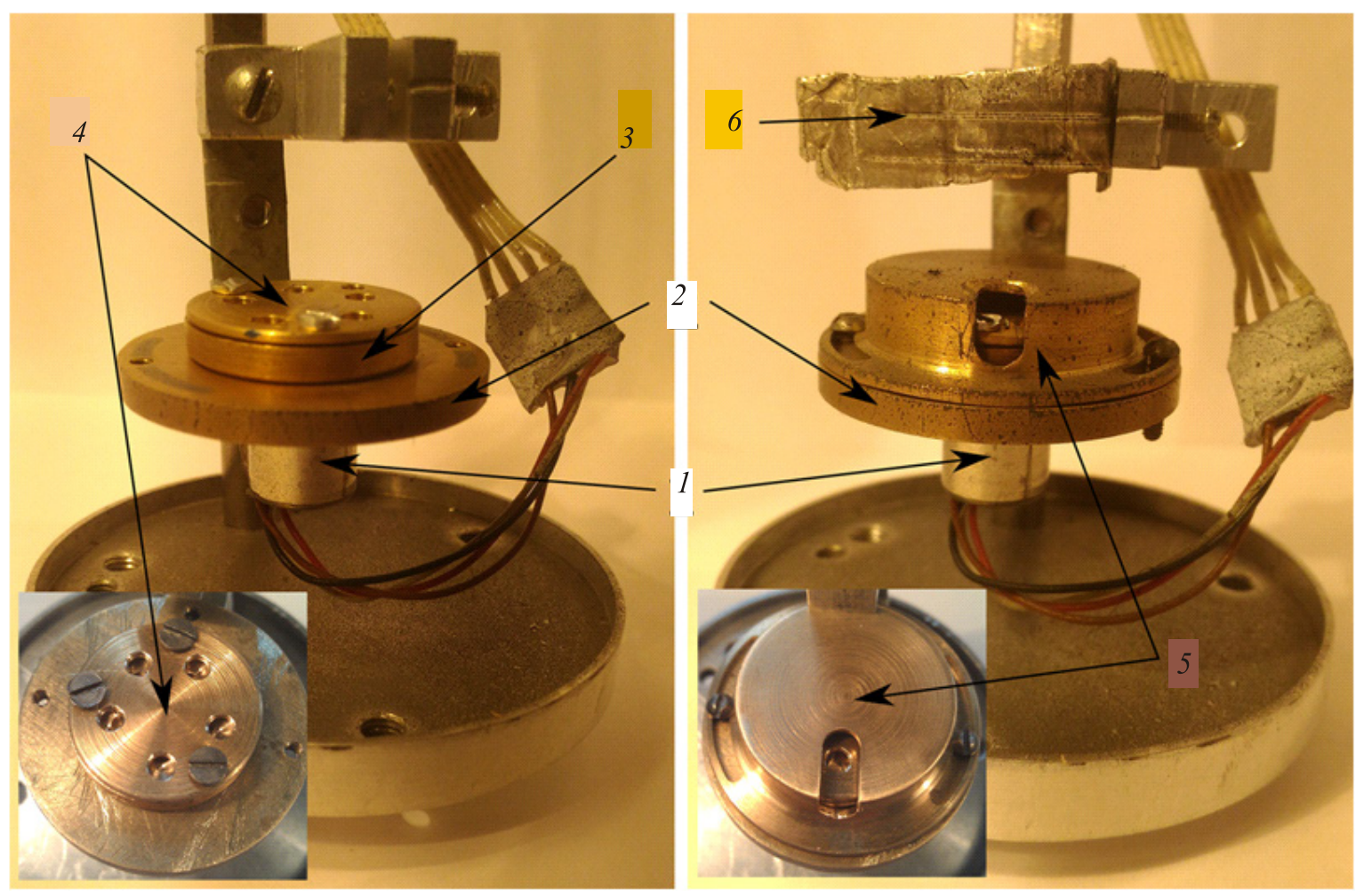

Fig. 4. The tool for samples exchange: 1 - stepper motor; 2 - support plate; 3 - turret; 4 - disk; 5 - cap; 6 - target. 
The discovering [31] and then explanation by the development of the Rayleigh instability [32] of the decay at low temperature of thin nanowires gave rise another hypothesis about the origin of the nanometer spheres, that included in the nanowires. According to it, these inclusions did not exist initially, and they were formed during heating of the samples to room temperature prior to the electron microscopy studies, as a consequence of Rayleigh instability, which leads, as is known, to bean pod shape.

If so the ratio between the number of small and large clusters should have changed under change of the laser power density, and we were hoping to see this in the relevant experiments. The results of our preliminary measurements was obscure, so we have decided with the help of a new technique to prepare several samples at different distances $X$ from the focusing lens to the target in the same experiment.

A convergence angle of the laser beam after the lens measured by using IR visualizator allows to calculate the diameter of the laser spot on the target at any $X$. Up to the diameter of $100 \mu \mathrm{m}$ the size is practically independent on $X$, while after detuning for more then $6 \mathrm{~mm}$ the spot diameter is determined by the law of geometrical optics.

The example of Fig. 5 shows photomicrographs of indium nanowires obtained by using the stepper motor in the same experiment for two of the lens-target distances: 1) the distance with detuning of $6 \mathrm{~mm}$ when, as in a focus, the spot diameter is about $100 \mu \mathrm{m}$ (see Figs. 5 (a), (c), (e)), and 2) with detuning of $18 \mathrm{~mm}$ when the spot diameter is about $250 \mu \mathrm{m}$ and the density of laser irradiation is 6 times less

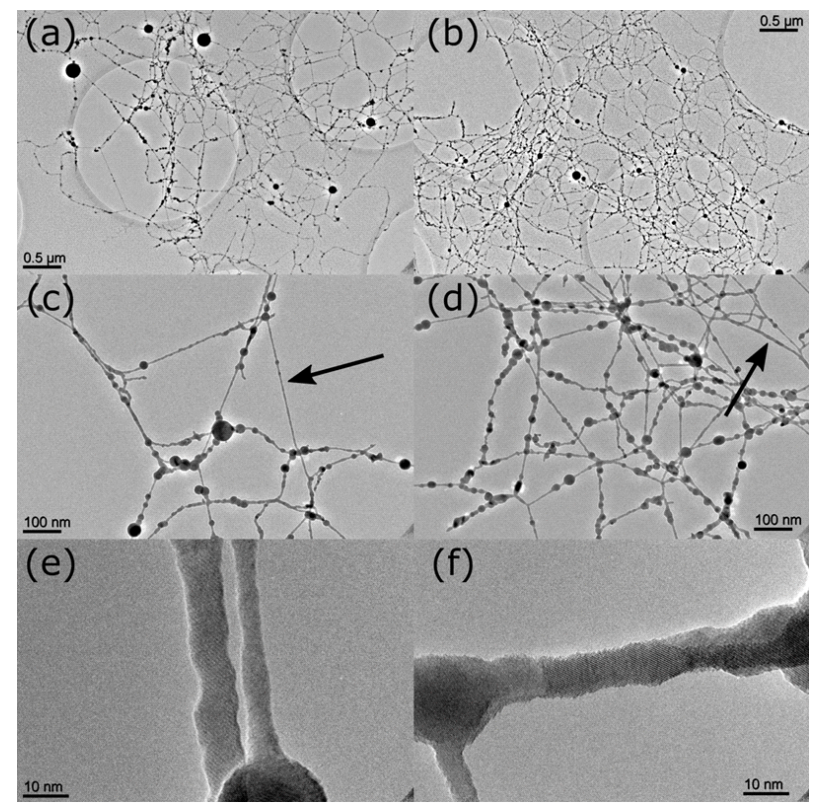

Fig. 5. TEM image of indium nanowires: (a), (c), (e) - morphology and structure of condensation products with decreasing of the distance from lens to target by $6 \mathrm{~mm}$; (b), (d), (f) - with decreasing of the distance by $18 \mathrm{~mm}$. (see Figs. 5 (b), (d), (f)), the duration of laser irradiation in the 1 st case has been more than doubled in comparing with the 2 nd case).

At the defocusing corresponding to spot size of $450 \mu \mathrm{m}$ at 3 times longer exposure neither nanowires nor spherical clusters were not detected on the grid, although the visible plasma radiation in the laser spot has been still observed.

These experiments indicate that above the ablation threshold the reduction of laser power density changes the output of nanoproducts but does not change significantly neither their morphology nor structure (though the number of large spheres seems to be less at low laser light density). This is indication that the spherical nanoclusters are nucleated not in plasma but later in liquid helium. It is important that there are some long fragments among the nanowires where spherical inclusions are absolutely absent (they are marked with arrows in the figure). This means that these spherical inclusions do not occur during the wires postheating, and it will be possible to get rid of them, in particular by changing of the vortices concentration by manipulating the rotation rate of the target or of metal density in helium by applying a femtosecond laser.

\subsection{The continuous rotation of target}

As in the case of stepper motors under selection of motors for target rotation, we have been guided by considerations of miniature size and lack of plastic in rotating parts. But here, for obvious reasons, an operational life of the motor, a relatively high speed and low power consumption bring into the foreground. At the same time, while testing we took in mind that the motor power consumption in superfluid helium may be much lower than under normal conditions because of the sharp fall of motor's winding ohmic resistance with decreasing of the temperature. The several types of micromotors shown on Fig. 6 have been tested.

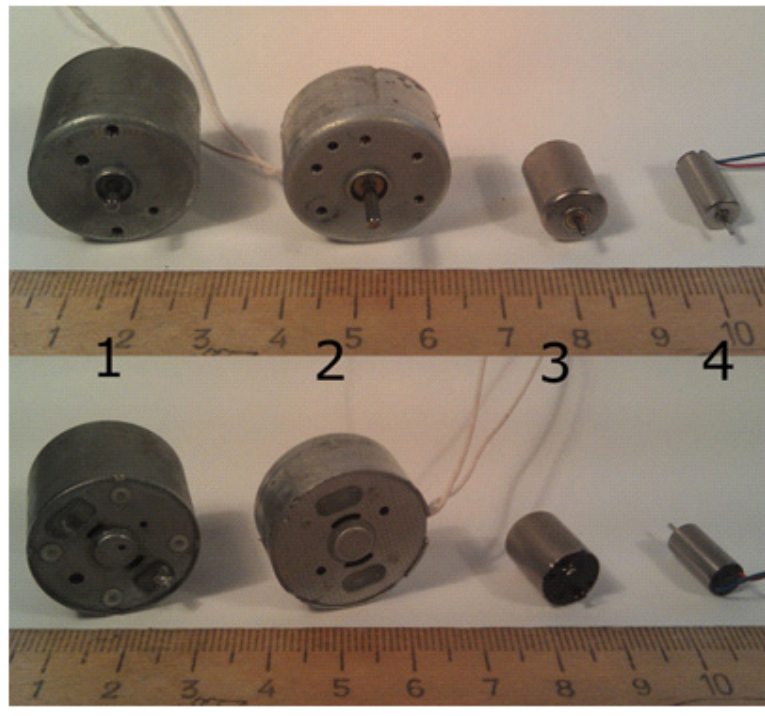

Fig. 6. The motors under testing (views from two sides). 
As the stepper motors, they rotated for a long time in ethanol for removing liquid lubricant which then was replaced by molybdenum disulfide. Then the motors were tested in liquid nitrogen. Motors No. 1 and No. 2 have worked in liquid nitrogen but motors No. 3 and No. 4 ceased work there. Because at the same frequency of rotation the power consumption of motor No. 2 was lower than that of the motor No. 1, the helium experiments were carried out with the motor No. 2. It was a collector bipolar dc motor with three sleeves on the rotor and two brushes. Its characteristics were: operating voltage of 1.5-6 V, speed of 3500-7000 rev/min.

Motor rotation frequency dependence on power consumption for the air and liquid nitrogen temperatures is shown in Fig. 7. It can be seen from the figure that with the using of solid lubricant rotational speed was proportional to power consumption up to the rates of about 40 revolutions per second. According to the above estimation it was enough for the density of quantum vortices generated by the rotation to be twice higher than the density of that created by the laser, even with the laser pulse repetition rate of $4 \mathrm{kHz}$. A positive moment was that given speed in liquid nitrogen was achieved at significantly lower power than in ambient conditions, which we have attribute to the decrease in the ohmic resistance of the windings. The negative feature was the appearance at $77 \mathrm{~K}$ of voltage threshold below which the rotation of the shaft didn't start, though above it the shaft rotation started at once with a frequency of several tens of hertz (see Fig. 7).

The experiments in superfluid helium have showed that at a temperature of about $1.6 \mathrm{~K}$ motor behavior was unstable, the rotation was accompanied by jerks. Anyway it works in He II for approximately one hour and we have managed to perform the experiment on indium nanowire production with rotating target. After that it stopped to work irreversibly, and the disassembly has shown that the cause of jerks and rubbing was, apparently, the collector-brush assembly — brass brushes of the motor were strongly deformed.

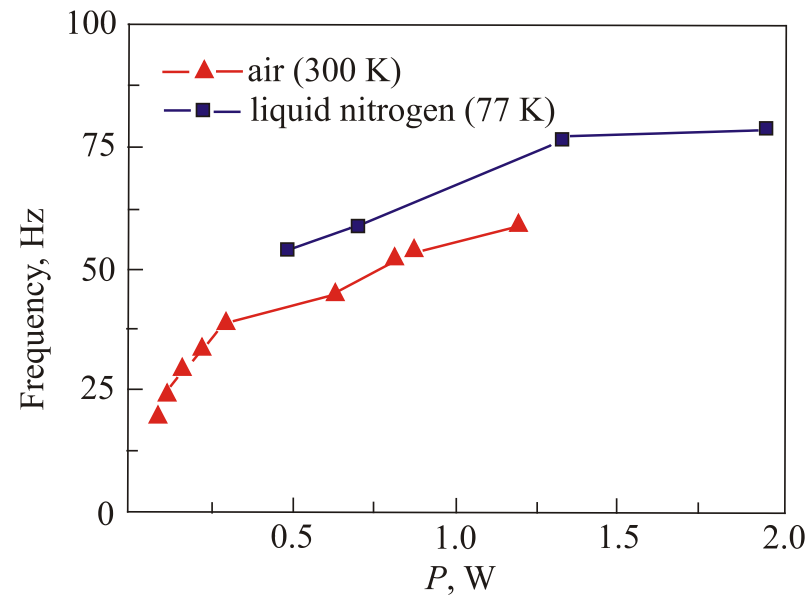

Fig. 7. Dependence of motor rotational velocity on the power consumption.
We see as the reason for the failure the application of the motor with brushes. At the temperature of liquid helium molybdenum disulfide becomes insulating and the problem with electrical contact of the collector with brushes arises. Highspeed brushless micromotors exists, and we hope to use them to overcome incipient problems.

\section{Conclusion}

Nobody intentionally developed low-power electric motors capable to operate at low temperatures in liquid helium. But the action of electric micromotors is based on the physical principles, which work only better with decreasing temperature. At the same time, the number of daily tasks for their application is so large and, accordingly, the variety of motor types, designed to solve them is so rich that you can try to choose that of them which by construction materials, size, capacity and other characteristics are able to carry out necessary movements inside the cryostat. In our case, their use has led to a significant increase in performance of scientific work, and we hope that in the near future we will solve with the using electric motors the problems that are not fully resolved now. We believe that our experience can be useful for a wide range of experimenters working in the field of lowtemperature physics, and perhaps not only for them.

\section{Acknowledgments}

The authors are grateful to S.B. Echmaev for the technical aid and to I.I. Khodos for the TEM study.

This work was supported by the Russian Science Foundation (RSF), Project \#14-13-00574.

1. R.J. Donnelly, Quantized Vortices in Helium II, Cambridge University Press, Cambridge (1991).

2. E.B. Gordon, A.V. Karabulin, V.I. Matyushenko, V.D. Sizov, and I.I. Khodos, Fiz. Nizk. Temp. 36, 740 (2010) [Low. Temp. Phys. 36, 590 (2010)].

3. P. Moroshkin, V. Lebedev, B. Grobety, C. Neururer, E.B. Gordon, and A. Weis, EPL 90, 34002 (2010).

4. E.B. Gordon, A.V. Karabulin, V.I. Matyushenko, V.D. Sizov, and I.I. Khodos, J. Low. Temp. Phys. 172, 94 (2013).

5. A.V. Simakin, V.V. Voronov, N.A. Kirichenko, and G.A. Shafeev, Appl. Phys. A 79, 1127 (2004).

6. G.W. Yang, Prog. Mater. Sci. 52, 648 (2007).

7. H.B. Zeng, X.W. Du, S.C. Singh, S.A. Kulinich, S.K. Yang, J.P. He, and W.P. Cai, Adv. Funct. Mater. 22, 1333 (2012).

8. Z.P. Xu, Q.H. Zeng, G.Q. Lu, and A.B. Yu, Chem. Eng. Sci. 61, 1027 (2006).

9. M.J. Hajipour, K.M. Fromm, A.A. Ashkarran, D.J. de Aberasturi, I.R. de Larramendi, T. Rojo, V. Serpooshan, W.J. Parak, and M. Mahmoudi, Trends Biotechnol. 30, 499 (2012).

10. A.T. Bell, Science 299, 1688 (2003).

11. V. Polshettiwar and R.S. Varma, Green Chem. 12, 743 (2010). 
12. X.L. Luo, A. Morrin, A.J. Killard, and M.R. Smyth, Electroanal. 18, 319 (2006).

13. J.N. Anker, W.P. Hall, O. Lyandres, N.C. Shah, J. Zhao, and R.P. Van Duyne, Nature Mater. 7, 442 (2008).

14. H. Sankur and J.T. Cheung, Appl. Phys. A 47, 271 (1988).

15. O. Auciello, J. Emerick, J. Duarte, and A. Illingworth, J. Vac. Sci. Technol. A 11, 267 (1993).

16. E.B. Gordon, A.V. Karabulin, V.I. Matyushenko, V.D. Sizov, and I.I. Khodos, Las. Phys. Lett. 12, 096002 (2015).

17. D.O. Edwards, R.L. Kindler, and S.Y. Shen, Rev. Sci. Instrum. 46, 108 (1975).

18. I.R. Walker, Rev. Sci. Instrum. 64, 814 (1993).

19. F.S. Porter, S.R. Bandler, C. Enss, R.E. Lanou, H.J. Marls, T. More, and G.M. Seidel, Physica C 194, 152 (1994).

20. C. Guthmann, S. Balibar, E. Chevalier, E. Rolley, J.C. Sutra Fourcade, Rev. Sci. Instrum. 65, 273 (1994).

21. E. Popov and J. Eloranta, J. Chem. Phys. 142, 204704 (2015).

22. H.E. Hall, J.R. Hook, S. Wang, A.J. Armstrong, and T.D. Bevan, Physica B: Condens. Matter 41, 194 (1994).

23. V. Kovacik, M. Fukuda, T. Igarashi, K. Torizuka, M.K. Zalalutdinov, and M. Kubota, J. Low Temp. Phys. 101, 567 (1995).
24. E.B. Gordon, A.V. Karabulin, V.I. Matyushenko, V.D. Sizov, and I.I. Khodos, Chem. Phys. Lett. 64, 519 (2012).

25. E.B. Gordon, A.V. Karabulin, A.A. Morozov, V.I. Matyushenko, V.D. Sizov, and I.I. Khodos, J. Phys. Chem. Lett. 5, 1072 (2014).

26. E.B. Gordon, M.I. Kulish, A.V. Karabulin, and V.I. Matyushenko, Fiz. Nizk. Temp. 43, No. 10 (2017) [Low Temp. Phys. 43, No. 10 (2017)].

27. S.K. Nemirovskii, Phys. Rep. 524, 85 (2013).

28. R.J. Donelly, C.F. Barenghi, J. Phys. Chem. Ref. Data 27, 1217 (1998).

29. http://www.ebay.com/itm/272142563837

30. E.B. Gordon, A.V. Karabulin, V.I. Matyushenko, V.D. Sizov, and I.I. Khodos, J. Exp. Theor. Phys. 112, 1061 (2011).

31. E.B. Gordon, A.V. Karabulin, V.I. Matyushenko, V.D. Sizov, and I.I. Khodos, Phys. Chem. Chem. Phys. 16, 25229 (2014).

32. A. Volk, D. Knez, P. Thaler, A.W. Hauser, W. Grogger, F. Hofer, and W.E. Ernst, Phys. Chem. Chem. Phys. 17, 24570 (2015). 